\title{
Imaging spectropolarimetry for magnetic field diagnostics in solar prominences
}

\author{
R. Di Campli ${ }^{1,2}$, R. Ramelli ${ }^{1}$, M. Bianda ${ }^{1}$, I. Furno ${ }^{2}$, S. Kumar Dhara ${ }^{1}$, and L. Belluzzi ${ }^{1,3}$ \\ ${ }^{1}$ Istituto Ricerche Solari Locarno (IRSOL), Università della Svizzera italiana, 6605 Locarno, Switzerland \\ e-mail: mbianda@irsol.ch \\ 2 École Polytechnique Fédérale de Lausanne (EPFL), Swiss Plasma Center (SPC), 1015 Lausanne, Switzerland \\ 3 Leibniz-Institut für Sonnenphysik (KIS), 79104 Freiburg, Germany
}

Received 11 March 2020 / Accepted 12 October 2020

\begin{abstract}
Context. Narrowband imaging spectropolarimetry is one of the most powerful tools available to infer information about the intensity and topology of the magnetic fields present in extended plasma structures in the solar atmosphere.

Aims. We describe the instrumental set-up and the observing procedure that we have developed and optimized at the Istituto Ricerche Solari Locarno in order to perform imaging spectropolarimetry. A measurement that highlights the potential of the ensuing observations for magnetic field diagnostics in solar prominences is presented.

Methods. Monochromatic images of solar prominences were obtained by combining a tunable narrowband filter, based on two FabryPerot etalons, with a Czerny-Turner spectrograph. Linear and circular polarization were measured at every pixel of the monochromatic image with the Zurich Imaging Polarimeter, ZIMPOL. A wavelength scan was performed across the profile of the considered spectral line. The HAZEL inversion code was applied to the observed Stokes profiles to infer a series of physical properties of the observed structure.

Results. We carried out a spectropolarimetric observation of a prominence, consisting of a set of quasi-monochromatic images across the $\mathrm{He}$ I $\mathrm{D}_{3}$ line at $5876 \AA$ in the four Stokes parameters. The map of observed Stokes profiles was inverted with HAZEL, finding magnetic fields with intensities between 15 and $30 \mathrm{G}$ and directed along the spine of the prominence, which is in agreement with the results of previous works.
\end{abstract}

Key words. magnetic fields - techniques: imaging spectroscopy - techniques: polarimetric - instrumentation: polarimeters Sun: filaments, prominences

\section{Introduction}

The polarization of spectral line radiation contains a wealth of information about the physical and geometrical properties of the emitting plasma. Powerful diagnostic techniques, which exploit the theoretical interpretation of spectropolarimetric observations, have been developed and successfully applied in order to investigate the solar atmosphere and, in particular, its magnetism (e.g., Stenflo 1994; Landi Degl'Innocenti \& Landolfi 2004; del Toro Iniesta 2007). During the last decades, particular efforts, both from a theoretical and observational point of view, have been devoted to the diagnostics of the magnetic fields in solar prominences, exploiting the combined action of the Hanle and Zeeman effects in particular spectral lines, such as $\mathrm{He}_{\mathrm{I}} \mathrm{D}_{3}$ at $5876 \AA$ and He I $10830 \AA$ (Sahal-Brechot et al. 1977; Bommier \& Sahal-Brechot 1978; Landi Degl'Innocenti 1982; Bommier et al. 1994; Lin et al. 1998; Trujillo Bueno et al. 2002; Casini et al. 2003; Merenda et al. 2006; Ramelli et al. 2006; Martínez González et al. 2012, and references therein). A well-experimented technique to perform spectropolarimetric measurements in solar prominences is to combine a slitspectrograph with a polarimeter (Leroy et al. 1977; Bommier et al. 1994; Paletou et al. 2001). With this set-up, Stokes profiles can be measured at every point along the spectrograph slit (i.e., on a one-dimensional field-of-view). At the Istituto Ricerche Solari Locarno (IRSOL), measurements of polarization in solar prominences and spicules in the He I $\mathrm{D}_{3}$ multiplet have been performed with the slit-spectrograph configuration. The Stokes profiles were inverted using the HAZEL code, and magnetic field strengths between $20 \mathrm{G}$ and $40 \mathrm{G}$ were found (Ramelli et al. 2011, 2006; Ramelli \& Bianda 2005).

Several investigations of the magnetic fields in solar prominences using a two-dimensional (2D) map of Stokes profiles have been carried out in recent years. We mention here the work by Casini et al. (2003), who used a slit-spectrograph and performed a scan over the prominence by changing the slit position. The observations were carried out at the Dunn Solar Telescope. A map of the magnetic field was obtained by applying an inversion method based on principal component analysis (Casini et al. 2005). The inferred magnetic field was mainly horizontal with a strength between $10 \mathrm{G}$ and $20 \mathrm{G}$.

More recently, Orozco Suárez et al. (2014) obtained a map of the magnetic field in prominence threads, using the same slitscanning technique with the Tenerife Infrared Polarimeter (TIP) at the German Vacuum Tower Telescope (VTT) at the Observatorio del Teide (Spain). Although this method provides high spectral resolution $(11 \mathrm{~m} \AA$ ), the long time lapse between the measurements with different slit positions (the scan of the whole field-of-view took approximately $1.5 \mathrm{~h}$ ) may potentially affect the spatial coherence of the measurement. Interestingly, this possible drawback was not encountered by Martínez González et al. (2015), who observed a quiescent prominence by applying the 


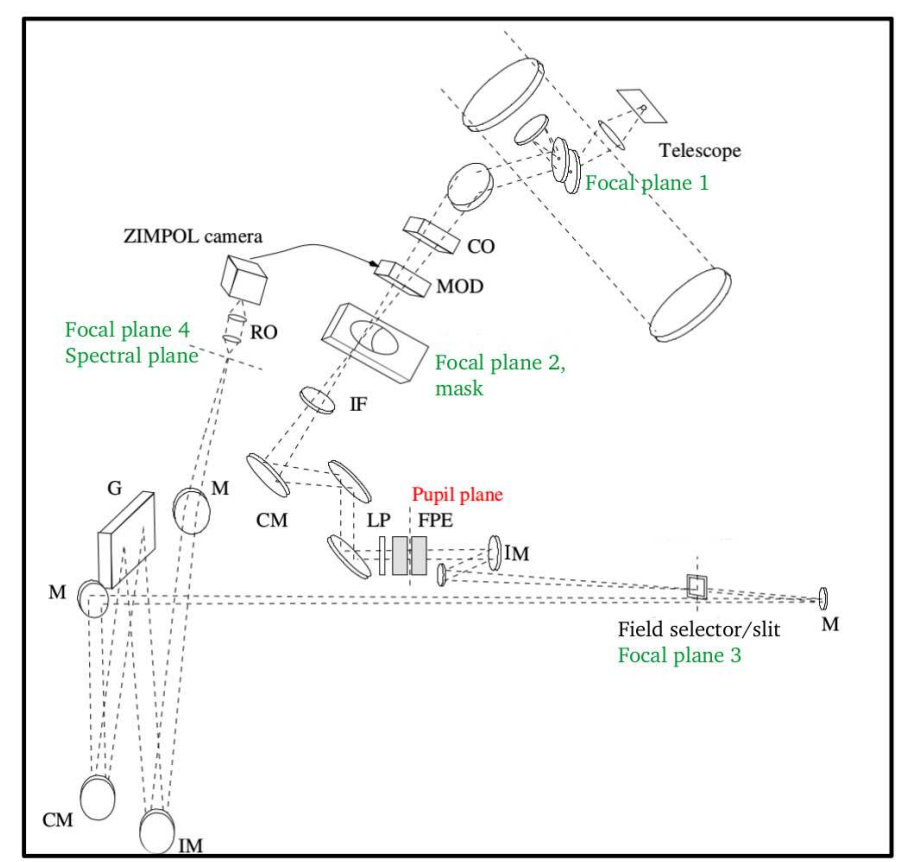

Fig. 1. Instrumental set-up. A tunable narrowband imaging filter is obtained by coupling two Fabry-Perot etalons (FPE) with a high resolution spectrometer based on a reflection grating $(\mathrm{G})$. The set-up includes the following: calibration optics (CO), a modulator (MOD), an interferometric pre-filter (IF), flat mirrors (M), collimating mirrors (CM), imaging mirrors (IM), a linear polarizer (LP), and reduction optics (RO).

slit-scanning technique and obtained consistent results on four consecutive scans, each lasting $30 \mathrm{~min}$.

In this paper, we present the instrumental set-up and the observing procedure that we have developed and optimized at IRSOL in order to perform imaging spectropolarimetry in solar prominences. Imaging observations were obtained with a voltage-tunable narrowband filter based on Fabry-Perot etalons (FPE). The polarimetric analysis was performed with the Zurich IMaging POLarimeter (ZIMPOL). The proposed observing technique has a very promising diagnostic potential as it provides spatially-coherent maps of the magnetic field vector, although it somehow sacrifices the temporal coherence.

The instrumental set-up is described in Sect. 2. The observing procedure and the data reduction technique are discussed in Sect. 3, where particular attention is paid to the characterization and correction of instrumental polarization as well as to the subtraction of stray light. In Sect. 4, we provide a detailed description of an observation of a prominence in the $\mathrm{He} \mathrm{I}_{3}$ line, and we highlight its diagnostic potential by showing the map of the magnetic field vector that has been obtained from the inversion of the observed Stokes profiles. The inversion was performed with a two-dimensional distribution of the HAZEL code (Asensio Ramos et al. 2008). Concluding remarks are provided in Sect. 5.

\section{Instrumentation}

The observations were performed with the $45 \mathrm{~cm}$ aperture Gregory-Coudé telescope of the Istituto Ricerche Solari Locarno (IRSOL). A schematic layout of the instrumental set-up is shown in Fig. 1. A narrowband imaging filter was obtained by combining two voltage-tunable $\mathrm{LiNbO}_{3}$ FPEs and a highresolution Czerny-Turner spectrograph (for details see Kleint et al. 2011). Polarization measurements were carried out with ZIMPOL (Ramelli et al. 2010), which allows for one to reach polarimetric sensitivities on the order of $10^{-4}$. This set-up allows one to perform quasi-monochromatic ( $35 \mathrm{~m} \AA$ spectral width) full-Stokes imaging polarimetry on a field-of-view (FOV) of about $65^{\prime \prime} \times 195^{\prime \prime}$. This configuration exploits the spectrograph grating in order to select the FPE channels, thus allowing for observations in any spectral line from $390 \mathrm{~nm}$ to $660 \mathrm{~nm}$ without the need for dedicated narrowband pre-filters.

The existing FPE set-up (see Kleint et al. 2011) was modified considering that prominences are observed off-limb. The main changes are the following: (a) the de-rotator was abandoned to reduce spurious effects and increase the instrument transmittance; and (b) the photoelastic modulator (MOD in Fig. 1) was replaced by a modulator based on two ferroelectric liquid crystal retarders (FLC; Gisler et al. 2003). This modulator, which was build following the principles described in Gisler et al. (2003), allows for a full-Stokes analysis and can perform high frequency ( $1 \mathrm{kHz}$ ) modulation to avoid spurious seeing-induced cross-talks between different Stokes parameters. A synchronous demodulation was performed with the ZIMPOL camera, which has a masked CCD sensor equipped with cylindrical microlenses (for details see Ramelli et al. 2010).

The absence of the optical de-rotator implies a rotation of the solar image. Since IRSOL's telescope has an equatorial mount, the image rotates with respect to the sensor with a constant angular speed. This rotation is numerically compensated in the data reduction as described in Sect. 3.

Reflections between internal optical components in the double FLC modulator can produce instrumental polarization and fringes. The intensity of the fringes is maximal when the modulator is perfectly perpendicular to the beam. By tilting the modulator, the amplitude of fringes decreases below $10^{-4}$ in fractional polarization. Internal reflections in the modulator also generate a secondary (ghost) image of the solar disk, which is projected on the sensor. Since these ghost image beams are not modulated with the same scheme as the principal beam, they are a possible source of spurious polarization signals. Figure 2 shows the instrumental polarization, which results in a polarized band localized near the limb. The four measurements shown in panels a-d of Fig. 2 were performed with different orientations of the solar limb on the modulator. From the observations, we deduce that the ghost image of the disk is shifted perpendicularly to the tilt axis of the modulator, which is vertical on the optical bench. The amplitude of the instrumental polarization is on the order of $1 \%$, which is, in the worst case, one order of magnitude higher than the expected fractional polarization signal we want to measure. The tilt angle of the modulator must be carefully chosen to balance these two effects (interference fringes and internal polarizing reflections). To minimize the width of the band affected by the disk image reflection, the modulator was set as close to the focal plane as possible, thus minimizing the shift between the primary and the secondary images.

A motorized mask covering the solar photosphere was installed in the focal plane (see Fig. 1) to avoid the saturation of the sensor on the disk ${ }^{1}$. This allows for an increase in the exposure time of a single frame, thus improving the signal-to-noise ratio in off-limb observations. The mask rotates with an angular speed that matches the image rotation.

\footnotetext{
1 We note that the ZIMPOL technique allows one to fix a high modulation and demodulation frequency (in these measurements $1 \mathrm{kHz}$ ), independently of the exposure time.
} 


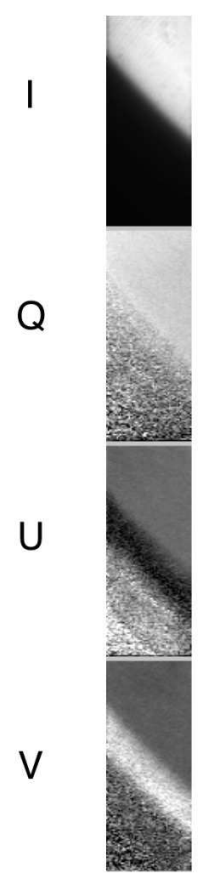

(a)

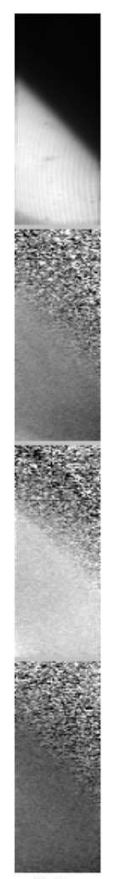

(b)

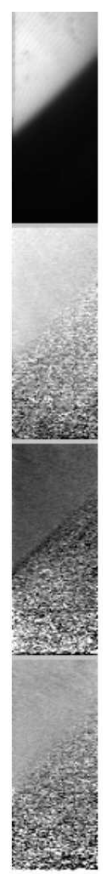

(c)

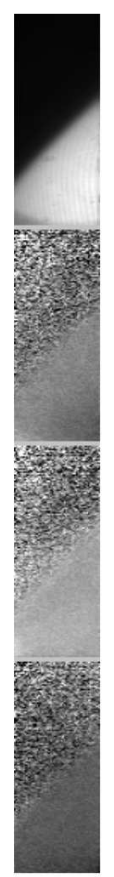

(d)
Fig. 2. Panels $a-d$ : four measurements with different limb orientations on the modulator. The intensity image $I$ allows one to see the limb position. In the polarization images, artifacts due to the ghost images (originated by the solar disk) are seen as a band immediately above the solar limb. These are more evident in column $(a)$ where the modulator is tilted and placed in an unfavorable position. The field of view is $65^{\prime \prime} \times 195^{\prime \prime}$.

The FPE spectral channel wavelength depends on the incidence angle of the light, as described by Kleint et al. (2011). Since FPEs are in a collimated set-up, beam bundles originating from distinct image points have different incidence angles on the étalons and, therefore, different transmitted wavelengths. This effect, called blue-shift, can be measured and Stokes profiles were corrected accordingly, as described in Sect. 3.

Another instrumental effect that needs to be corrected is image deformation. This is mainly attributed to the following two sources: anamorphic reflection on the grating and the asymmetric sampling of sensor pixels (Kleint et al. 2011). In fact, the ZIMPOL demodulation technique requires one to mask three out of four pixel rows. Since the grating is illuminated by a collimated beam, distinct image points have a different incidence angle on the grating. In addition, the reflection angle is different from the incidence angle, generating anamorphic reflection. To compensate for the deformation introduced by the sensor masking and anamorphic reflection, the image array is horizontally binned to restore correct proportions.

\section{Observing procedure and data reduction}

Many test measurements, which were performed between February and August 2017, allowed us to define an optimized observing procedure and data reduction. Before starting the observation and performing the spectral scan of the chosen spectral line, it was necessary to perform an accurate wavelength tuning of the spectrograph and of the FPE. This procedure permits one to calculate the voltages at which the FPEs have to be set in order to select the desired wavelength at a given temperature. To do so, we first obtained a reference spectrum by placing a slit at focal plane 3 (see Fig. 1) with the two FPEs out of the beam.
The obtained spectra were then cross-correlated with the FTS atlas (Kurucz et al. 1984). Subsequently, the FPEs were inserted into the beam and tuning was performed by changing the voltage. We note that the second FPE was tilted by about $0.1^{\circ}$ with respect to the optical axis to avoid ghost images on the camera, which are produced by internal reflections of the interferometers (for details see Kleint et al. 2011). The resulting transmission spectra provide the required values in order to tune the FPEs.

The polarization calibration measurements were obtained with respect to a Stokes reference system fixed with the observatory, where the Stokes $+Q$ was chosen to lay on the vertical plane along the beam at focus 2 . The linear polarization calibration measurements were carried out with a linear polarizer, which was inserted before the FLC modulator, that is to say rotated in four different positions corresponding to $+Q,+U,-Q$, and $-U$. The two circular polarization calibration measurements $(+V,-V)$ were obtained by adding a quarter-wave plate retarder.

The observation consists of a series of $2 \mathrm{D}$ monochromatic full-Stokes measurements performed at the wavelengths chosen to sample the considered spectral line. Such measurements were carried out one after the other with a given integration time. One measurement was also performed at a continuum wavelength (background image) to quantify the intensity and polarization contribution of the diffused light and of the continuum emission. The demodulation matrix determined with the calibration permits one to get a Stokes image that needs to be further reduced.

A standard flat fielding was carried out for each wavelength step by moving the telescope randomly around quiet regions close to the solar disk center approximatively every $30 \mathrm{~min}$. In order to take the constant image rotation $\left(15^{\circ} \mathrm{h}^{-1}\right)$ into account, the observed image was numerically rotated in order to have the limb oriented along a chosen direction (for instance the horizontal direction, as seen in Fig. 3). The Stokes $Q$ and $U$ axes were also rotated from the basis fixed to the observatory to a basis fixed to the solar image, so that $+Q$ was oriented parallel to the solar limb at the observed position.

As the modulator was set after the folding mirrors of the telescope, the instrumental polarization produced by the telescope itself generated cross-talks between the Stokes components. These were corrected for by considering that the Müller matrix of the telescope is almost constant during one solar day since it only depends on the declination of the pointing direction.

The cross-talk from intensity to polarized components was corrected for by considering the mean offset from zero in Stokes $Q / I, U / I$, and $V / I$ obtained in the flat field measurements. To measure the linear to circular polarization cross-talk coefficients of the Müller matrix with a few percent precision, a linear polarizer was installed in front of the telescope at four different orientations $\left(0^{\circ},+45^{\circ}, 90^{\circ}\right.$, and $-45^{\circ}$ with respect to the hour circle). The circular to linear polarization cross-talk coefficients were determined considering the symmetries of the theoretical Müller matrix described by Sanchez Almeida et al. (1991).

The background measurement was then subtracted from the line scan measurements to eliminate the contribution from the continuum, and possibly some remaining spurious instrumental polarization. This technique isolates the signals coming from the selected atomic line of interest from the stray light. For each wavelength measurement, the background was rotated and rescaled by a factor of $n_{k, \lambda}$ (where $k$ indicates the Stokes component and $\lambda$ is the wavelength); this was determined so as to minimize the quantity

$\Delta I_{k, \lambda}=\sum_{i=1}^{N} \sum_{j=1}^{M}\left|S_{i, j, k, \lambda}-n_{k, \lambda} B_{i, j, k, \lambda}\right|$ 
where $N$ and $M$ are the numbers of the image rows and columns, the pairs of indices $(i, j)$ identify the considered pixel, $S$ is the signal, and $B$ is the background.

In order to measure the blue-shift surface generated by the FPE across the FOV of the image, a wavelength scan of a nearby absorption line was performed. A Gaussian fitting of the scanned absorption line profile was performed in order to determine the wavelength corresponding to the line center. For every spatial coordinate, the wavelength of the line center is different due to the blue-shift effect. This provides a "surface" representing the induced wavelength shift across the whole FOV, which can be fitted with a quadratic polynomial surface. The obtained blue shift across the FOV is on the order of a few $\mathrm{m} \AA$. The blue-shift effect can therefore be corrected for by shifting every Stokes profile by the amount corresponding to the considered pixel.

\section{Experimental results}

Following the observing technique described in Sect. 3, a prominence was successfully observed in the $\mathrm{He} \mathrm{I} \mathrm{D}_{3}$ line at $5876 \AA$. The prominence appeared on June 7, 2017 at the SE limb at a latitude of $48^{\circ}$. The sky was clear and the observing conditions were good. A scanning across the $\mathrm{He} \mathrm{I}_{3}$ line was performed considering a set of 16 wavelengths, from $5875.40 \AA$ to $5876.02 \AA$, with a step of $0.04 \AA$. In order to reduce the observing time interval with the aim of maintaining as much temporal coherence as possible, we decided to narrow the recorded spectral range so that it covers all the components of the $\mathrm{He} \mathrm{I} \mathrm{D}_{3}$ multiplet but not the whole line wings. For each wavelength, we acquired 12 frames, each with a $10 \mathrm{~s}$ integration time. The time sequence required $4 \mathrm{~min}$ to take a set of flatfield images (one flatfield image per wavelength), $26 \mathrm{~min}$ to take the images in the prominence, and again 4 min for the flatfield. Although more sequences were recorded, only one was used for this analysis. The reason is that we noticed that the results worsen when averaging over more sequences because of the lack of temporal coherency. A background image was obtained by performing a measurement in the continuum at $5874.2 \AA$. The instrumental blue-shift across the FOV (see Sect. 3) was determined by scanning the absorption profile of the nearby Nb I line at $5878 \AA$. Figure 3 shows the Stokes images of the prominence obtained at three different wavelength positions. The bright prominence structure is well observed outside the solar limb in the Stokes $I$ images. The Stokes $Q$ and $U$ images show clear polarization signals in the prominence structure. The reference direction for positive Stokes $Q$ was chosen to be parallel to the limb.

In order to increase the signal-to-noise ratio, the Stokes profiles were obtained by averaging groups of $3 \times 3$ pixels in the prominence region. Example profiles, which were normalized to the maximum value of the intensity, $I_{\max }$, are shown in Fig. 4. The zero of the wavelength axis was chosen at the position of the red component of the $\mathrm{He} \mathrm{I}_{3}$ multiplet (5875.97 $\AA$ ). First of all, we notice that the observed polarimetric profiles are consistent with those previously measured by Ramelli et al. (2006) using the spectrograph. A strong positive Stokes $Q$ signal is observed at the position of the blue component, indicating that the radiation is linearly polarized parallel to the limb. This signal is produced by the scattering of the anisotropic radiation field, which illuminates the He I atoms in the prominence (scattering polarization). A clear $Q$ signal is also produced by the red component, which has a higher polarizability. It must be observed that the amplitude and shape of the latter signal can appreciably change in different observations. Moreover, it has to be considered that

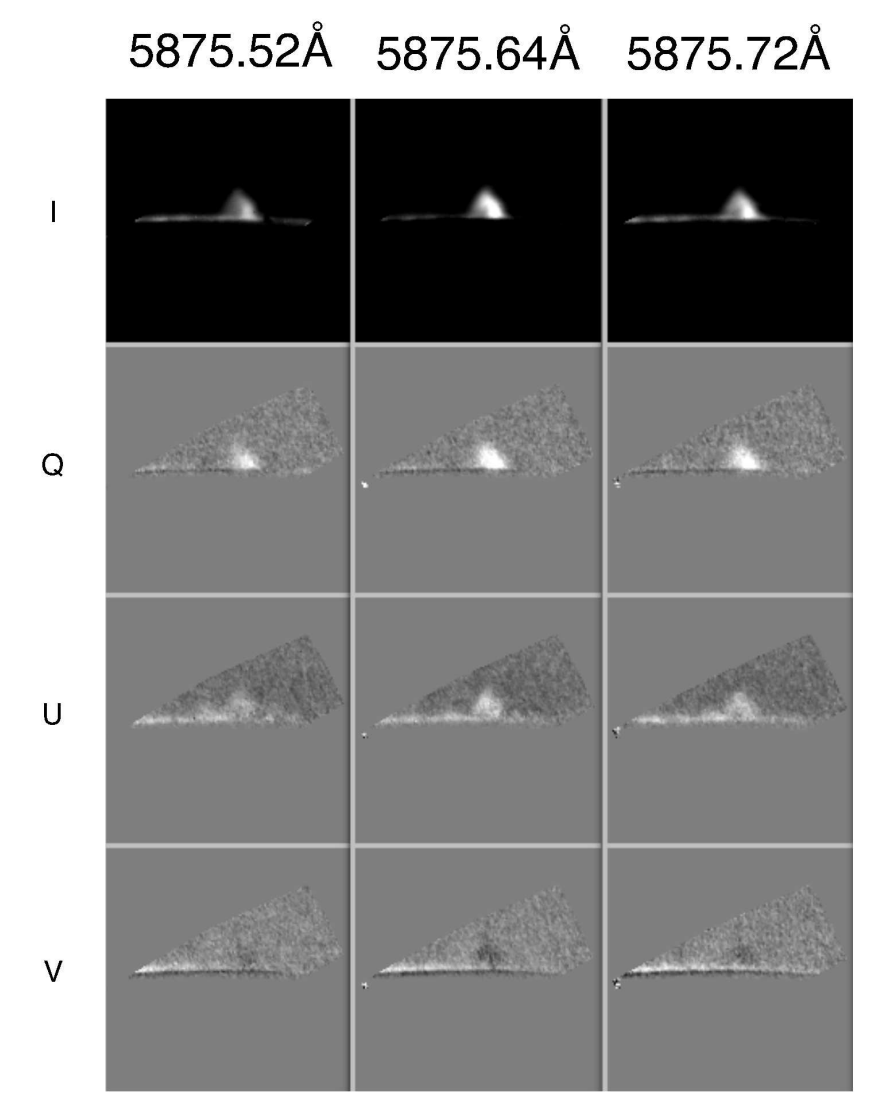

Fig. 3. Monochromatic images of the observed prominence at various wavelengths in the $\mathrm{He} \mathrm{I} \mathrm{D}_{3}$ line. The measurement was carried out on June 7, 2017. The structure of the prominence is well defined, and clear polarization signals are detected.

the lack of temporal coherency may somehow affect the quality of the profiles, following temporal changes and oscillations of the prominence. The longitudinal oscillations may generate Doppler effect variations on the order of $0.04 \AA$, which reduce the spectral resolution.

A small $U$ polarization signal is also visible. This is a signature of the Hanle effect (i.e., the magnetic-field-induced modification of line scattering polarization). Lastly, some net circular polarization is also appreciable, although the significance of this signal strongly depends on the accuracy of the cross-talk correction. If its solar origin is confirmed, such a net circular polarization could be produced through the so-called alignment-to-orientation conversion mechanism, which operates in the presence of magnetic fields in the incomplete PaschenBack effect regime (see Landi Degl'Innocenti 1982; Landi Degl'Innocenti \& Landolfi 2004). Indeed, it has been shown that this mechanism is particularly effective in the $\mathrm{He} \mathrm{I}_{3}$ line, in which the incomplete Paschen-Back effect regime is reached for magnetic fields on the order of $10 \mathrm{G}$ (see Landi Degl'Innocenti 1982; Landi Degl'Innocenti \& Landolfi 2004).

To estimate the magnetic field of the plasma structures in the observed solar prominence, an inversion of the measured Stokes profiles was performed using the advanced synthesis and inversion HAnle and ZEeman Light (HAZEL) code, developed by Asensio Ramos et al. (2008). This code is based on the quantum theory for the generation and transfer of polarized radiation described in Landi Degl'Innocenti \& Landolfi (2004). In particular, it accounts for the atomic polarization that is induced by anisotropic optical pumping processes, together with the impact 

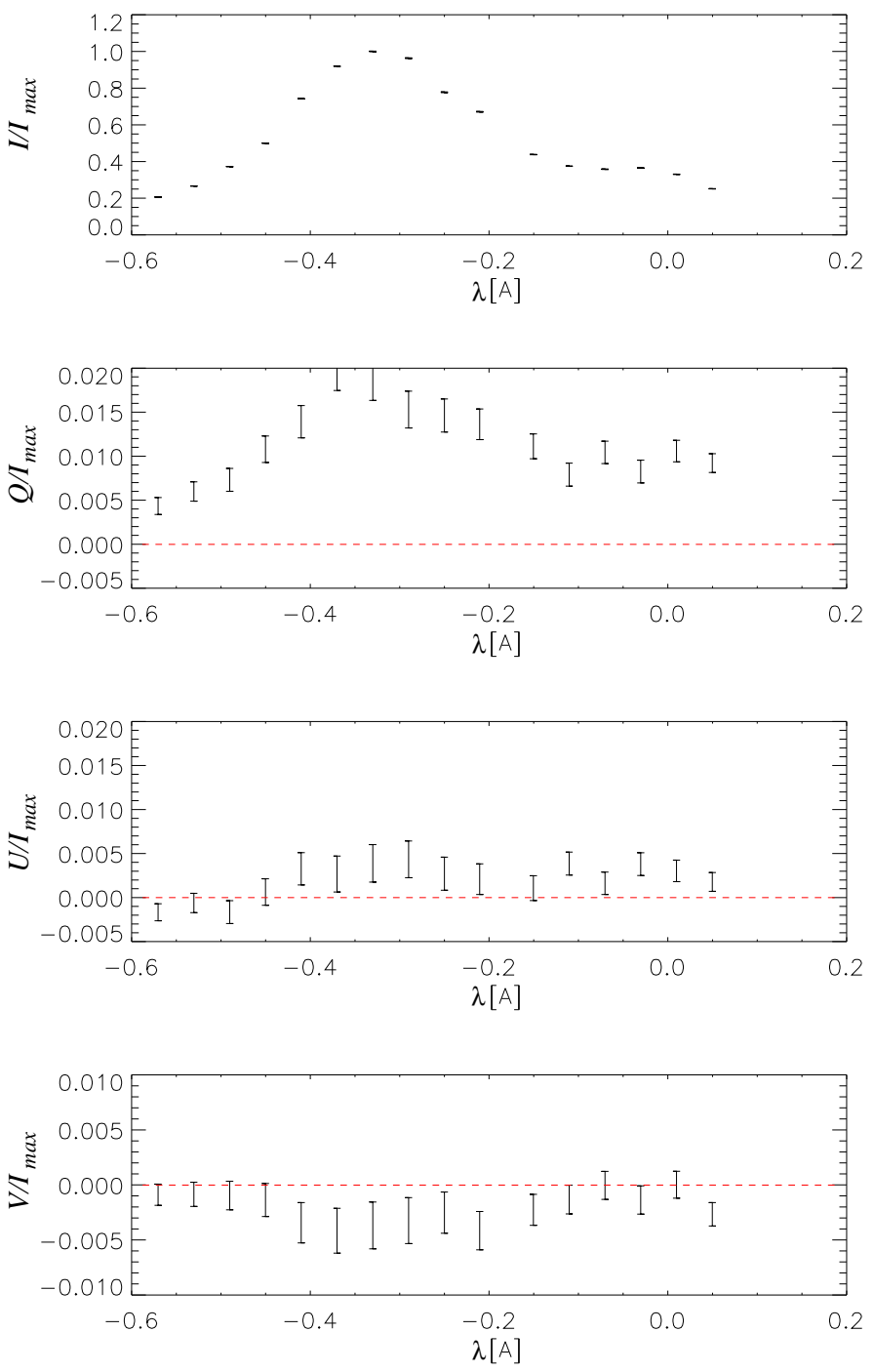

Fig. 4. Example of measured Stokes profiles, normalized to the maximum intensity, of the $\mathrm{He}$ I $\mathrm{D}_{3}$ line observed on the prominence. The zero of the wavelength scale is at $5875.97 \AA$. A significant positive Stokes $Q$ signal can be clearly observed. Weak $U$ and $V$ signals are also detected. However, the significance of the latter strongly depends on the accuracy of the cross-talk correction. The reference direction for positive Stokes $Q$ was chosen to be parallel to the solar limb. Error bars represent the statistical photon noise uncertainty.

of the magnetic field through the combined action of the Hanle and Zeeman effects. Magnetic fields of arbitrary strengths, from the Zeeman to the complete Paschen-Back effect regime, can be considered. The prominence is modeled as an optically thin, constant property slab of plasma. Stimulated emission processes are taken into account.

Figure 5 shows the magnetic field maps obtained from the inversion of the Stokes profiles. The average magnetic field strength in the prominence structure is found to be between 15 and $30 \mathrm{G}$. The direction of the magnetic field vector is mainly horizontal along the spine of the prominence. These results are coherent with those of previous studies carried out on quiescent prominences (e.g., Bommier et al. 1994; López Ariste \& Casini 2003; Casini et al. 2003). However, in some organized plasma structures of the observed quiescent prominence, the magnetic field strength is significantly larger (on the order of $45-50 \mathrm{G}$ ). We shall observe that we are not fully confident as to the reli-

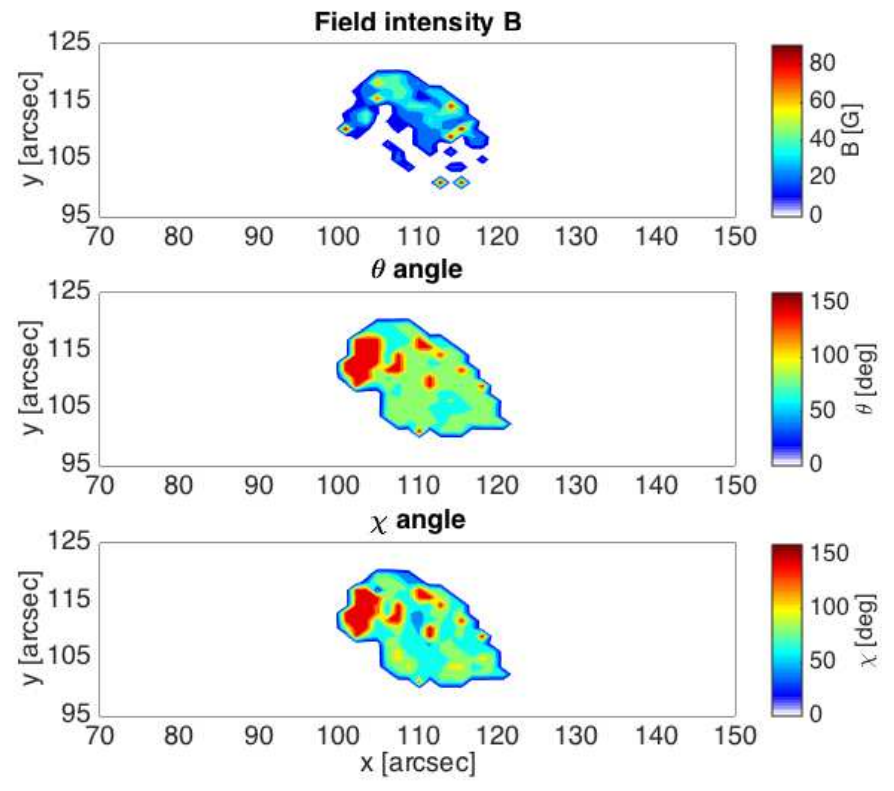

Fig. 5. Maps of the magnetic field in the prominence observed on June 7, 2017, obtained from the inversion of the four observed Stokes profiles of the $\mathrm{He} \mathrm{I}_{3}$ line. The maps show the intensity of the magnetic field (upper panel), the inclination $\theta_{\mathrm{B}}$ with respect to the local vertical (middle panel), and the azimuth $\chi_{\mathrm{B}}$, which was measured with respect to the projection of the line-of-sight on the plane parallel to the surface of the Sun (lower panel).

ability of the inversion at the spatial positions where the most intense magnetic fields are found. These results would require a more detailed analysis which, however, was not carried out given the merely illustrative purpose of this application. We also observe that although HAZEL finds spatially coherent parameters, intrinsic ambiguities (Hanle $180^{\circ}$, and Van Vleck) imply that this is not the unique possibility for the field configuration that could generate the observed Stokes profiles. More information on ambiguities in magnetic field diagnostics in solar prominences can be found in Merenda et al. (2006) and Casini et al. (2005).

\section{Conclusion}

Imaging spectro-polarimetry of solar prominences can be performed at IRSOL by combining the ZIMPOL polarimeter, a Czerny-Turner spectrograph, and two FPEs. We performed several days of test observations in order to understand and improve the quality of the data. This has allowed us to optimize the instrumental set-up, as well as the observing procedure and the data reduction technique. Instrumental polarization, image rotation, image deformation, FPE blue shifts across the FOV, stray light, and reflections have to be carefully compensated for to obtain the best possible Stokes profiles.

With the instrumental set-up and procedure described in this paper, on June 7, 2017, we carried out a 2D imaging spectropolarimetric measurement of a quiescent prominence in the $\mathrm{He}$ I $\mathrm{D}_{3}$ line at $5876 \AA$. Stokes profiles are obtained at every pixel of the image. The ZIMPOL polarimeter allows for the detection of weak polarization signals in the prominence. Using the HAZEL inversion code, it was possible to obtain 2D maps of the magnetic field vector of the prominence. We find predominantly horizontal magnetic fields, which are directed along the spine of the prominence, with strengths of about $15-30 \mathrm{G}$. These results are 
consistent with those of other investigations of quiescent prominences that were carried out in the past.

The observing technique that we have developed is very promising and deserves further attention. Indeed, reliable information on the strength and topology of the magnetic fields present in the observed prominence could be obtained from the inversion of the acquired spectropolarimetric data. These kinds of magnetic field diagnostics are essential to validate the multitude of theoretical and numerical models of solar prominences that have been proposed so far. Further work and better instrumentation have to be pursued, especially to increase the spectral and time resolution of the measurements.

Acknowledgements. IRSOL is supported by the Swiss Confederation (SERI) Canton Ticino, the city of Locarno and the local municipalities. This research work was financed by SNF 200020-169418 and 200020-184952. Daniel Gisler gave support for the new set-up and measurements. The development of the system to be adequate for working with the last version of the polarimeter, ZIMPOL3, was performed by Mathis Engelhardt (Fachhochschule RheinMain) as master thesis and by Damiano Kuthan.

\section{References}

Asensio Ramos, A., Trujillo Bueno, J., \& Landi Degl'Innocenti, E. 2008, ApJ, 683,542

Bommier, V., \& Sahal-Brechot, S. 1978, A\&A, 69, 57

Bommier, V., Landi Degl'Innocenti, E., Leroy, J.-L., \& Sahal-Brechot, S. 1994 Sol. Phys., 154, 231

Casini, R., López Ariste, A., Tomczyk, S., \& Lites, B. W. 2003, ApJ, 598, L67

Casini, R., Bevilacqua, R., \& López Ariste, A. 2005, ApJ, 622, 1265

del Toro Iniesta, J. C. 2007, Introduction to Spectropolarimetry (Cambridge: Cambridge University Press)
Gisler, D., Feller, A., \& Gandorfer, A. M. 2003, in Polarimetry in Astronomy, ed. S. Fineschi, Proc. SPIE, 4843, 45

Kleint, L., Feller, A., \& Gisler, D. 2011, A\&A, 529, A78

Kurucz, R. L., Furenlid, I., Brault, J., \& Testerman, L. 1984, Solar Flux Atlas from 296 to $1300 \mathrm{~nm}$ (New Mexico: National Solar Observatory)

Landi Degl'Innocenti, E. 1982, Sol. Phys., 79, 291

Landi Degl'Innocenti, E., \& Landolfi, M. 2004, Polarization in Spectral Lines (Dordrecht: Kluwer)

Leroy, J. L., Ratier, G., \& Bommier, V. 1977, A\&A, 54, 811

Lin, H., Penn, M. J., \& Kuhn, J. R. 1998, ApJ, 493, 978

López Ariste, A., \& Casini, R. 2003, ApJ, 582, L51

Martínez González, M. J., Asensio Ramos, A., Manso Sainz, R., Beck, C., \& Belluzzi, L. 2012, ApJ, 759, 16

Martínez González, M. J., Manso Sainz, R., Asensio Ramos, A., et al. 2015, ApJ, 802,3

Merenda, L., Trujillo Bueno, J., Landi Degl'Innocenti, E., \& Collados, M. 2006, ApJ, 642, 554

Orozco Suárez, D., Asensio Ramos, A., \& Trujillo Bueno, J. 2014, in Nature of Prominences and their Role in Space Weather, eds. B. Schmieder, J. M. Malherbe, \& S. T. Wu, IAU Symp., 300, 112

Paletou, F., López Ariste, A., Bommier, V., \& Semel, M. 2001, A\&A, 375, L39

Ramelli, R., \& Bianda, M. 2005, in Solar Magnetic Phenomena, eds. A Hanslmeier, A. Veronig, \& M. Messerotti, Astrophys. Space Sci. Lib., 320, 215

Ramelli, R., Bianda, M., Trujillo Bueno, J., et al. 2006, in Solar Polarization 4, eds. R. Casini, \& B. W. Lites, ASP Conf. Ser., 358, 471

Ramelli, R., Balemi, S., Bianda, M., et al. 2010, in Ground-based and Airborne Instrumentation for Astronomy III, Proc. SPIE, 7735, 77351Y

Ramelli, R., Trujillo Bueno, J., Bianda, M., \& Asensio Ramos, A. 2011, in Solar Polarization 6, eds. J. R. Kuhn, D. M. Harrington, H. Lin, et al., ASP Conf. Ser., 437, 109

Sahal-Brechot, S., Bommier, V., \& Leroy, J. L. 1977, A\&A, 59, 223

Sanchez Almeida, J., Martinez Pillet, V., \& Wittmann, A. D. 1991, Sol. Phys., 134, 1

Stenflo, J. O. 1994, Solar Magnetic Fields: Polarized Radiation Diagnostics (Dordrecht: Kluwer)

Trujillo Bueno, J., Landi Degl'Innocenti, E., Collados, M., Merenda, L., \& Manso Sainz, R. 2002, Nature, 415, 403 